\title{
SÍNDROMES DE DISPERSÃO DE SEMENTES EM TRÊS TRECHOS DE VEGETAÇÃO CILIAR (NASCENTE, MEIO E FOZ) AO LONGO DO RIO PINDAÍBA, MT ${ }^{1}$
}

\author{
Daniel Stefanello², Clarissa Fernandes-Bulhão ${ }^{3}$ e Sebastião Venâncio Martins ${ }^{4}$
}

\begin{abstract}
RESUMO - Este estudo foi desenvolvido visando identificar as proporções entre as síndromes de dispersão e os tipos de frutos encontrados em três áreas: nascente (Cerrado Rupestre), meio e foz (mata ciliar - Floresta Estacional Semidecidual) ao longo do Rio Pindaíba, MT. No trecho de nascente, 55,6\% das espécies amostradas eram zoocóricas, $43 \%$ anemocóricas e apenas $1,4 \%$ autocóricas, respectivamente. No trecho do meio, 85,7\% das espécies eram zoocóricas, $11,7 \%$ anemocóricas e apenas 1,3\% autocóricas. Na foz do Rio Pindaíba foram encontradas $77,5 \%$ de espécies zoocóricas, $20 \%$ de anemocóricas e apenas 2,5\% de autocóricas. A hipótese levantada neste estudo foi de que em áreas abertas (nascente) a proporção de espécies anemocóricas seria maior do que em ambientes fechados (meio e foz), onde predominaria a zoocoria. Entretanto, não houve diferença significativa nas proporções de espécies anemocóricas e zoocóricas na nascente (Cerrado Rupestre), enquanto no meio e na foz (mata ciliar) as proporções de zoocoria foram maiores. Ambientes florestais, estruturalmente mais complexos com menor circulação do vento e menor incidência luminosa, requerem estratégias de dispersão mais direcionadas e previsíveis como a zoocoria.
\end{abstract}

Palavras-chave: Dispersão de sementes, ecologia de sementes e mata ciliar.

\section{SEED DISPERSAL SYNDROMES IN THREE RIPARIAN VEGETATION SITES (SOURCE, MIDDLE AND ESTUARY) ALONG THE PINDAÍBA RIVER, MATO GROSSO STATE, BRAZIL}

\begin{abstract}
This study was carried out to identify the proportions between the dispersal syndromes and fruit types found in three regions: source (rupestrian cerrado), middle zone and estuary (riparian-semideciduous forest) along the River Pindaíba-MT. At the source, $55.6 \%$ of shrub-tree species are zoochoric, $43 \%$ are anemochoric and only $1.4 \%$ are autochoric. In the middle zone, $85.7 \%$ of species are zoochoric, $11.7 \%$ anemochoric and only $1.3 \%$ are autochoric. At the estuary of River Pindaiba, we found $77.5 \%$ of zoochoric species, $20 \%$ of anemochory and only $2.5 \%$ of autochory. Our hypothesis for the study was that, in open areas (source), the proportion of anemochoric species would be higher than in closed environments (middle zone and mouth), with the predominance of zoochory. However, there was no significant difference in the proportions of anemochoric and zoochoric species for the source (rupestrian cerrado), whereas in the middle zone and at the estuary(riparian), the proportions of zoochory were higher. Forest environments, which are structurally more complex with less wind circulation and lower light incidence, require dispersion strategies more directed and predictable, such as zoochory.
\end{abstract}

Keywords: Seed dispersal, seed ecology and riparian forest.

\section{INTRODUÇÃO}

A frutificação e a dispersão de sementes são etapas importantes do ciclo reprodutivo da maioria das plantas (GOTTSBERGER e SILBERBAUER-GOTTSBERGER,
1983). As diferentes maneiras como os diásporos são dispersos e a frequência com que atingem ambientes favoráveis para o estabelecimento da plântula é que determinam a riqueza e a distribuição espacial das populações de plantas (VAN DER PIJL, 1982).

\footnotetext{
${ }^{1}$ Recebido em 27-02-2008 e aceito para publicação em 23.06.2009.

${ }^{2}$ Programa de Pós-graduação em Ciência Florestal, Universidade Federal de Viçosa - Viçosa, MG. E-mail: <stefanello.daniel@ gmail.com>. ${ }^{3}$ Instituto de Ciências Naturais e Tecnológicas, Universidade do Estado do Mato Grosso- Nova Xavantina, MT. E-mail: <clarissafernandesb@yahoo.com.br>.

${ }^{4}$ Laboratório de Restauração Florestal, DEF, UFV, Bolsista Produtividade em Pesquisa do CNPq. E-mail: <venancio@ufv.br>
} 
Os padrões de distribuição espacial dependem de interações diretas e indiretas com as forças seletivas bióticas e abióticas da comunidade (TALORA e MORELLATO, 2000). Dessa forma, um dos processos que determinam a riqueza e distribuição das plantas é, sem dúvida, a síndrome de dispersão de frutos e sementes, como verificado por Pinheiro e Ribeiro (2001) e Peternelli et al. (2004).

A dispersão é caracterizada como o transporte dinâmico, resultando em estado passivo de distribuição dos diásporos desde a planta-mãe até um ambiente que ofereça condições favoráveis para o estabelecimento das espécies; assim, a dispersão torna-se realidade biológica que assegura a expansão das espécies (VAN DER PIJL, 1982).

O conjunto de processos pelos quais sementes e frutos são dispersos ou transportados, à maior ou menor distância da planta-mãe, é definido como síndrome de dispersão. Existem quatro principais grupos de síndromes: 1) Zoocoria - dispersão realizada por diferentes grupos de animais (GRAHAM et al., 1995); 2) Anemocoria - síndrome cujo principal agente dispersor é o vento (GOTTSBERGER e SILBERBAUER-GOTTSBERGER, 1983; HOWE e SMALLWOOD, 1982; VANDER PIJL, 1982); 3) Autocoria - as sementes são dispersas pelas próprias plantas, em que os frutos se abrem por deiscência explosiva e lançam as sementes (HOWE e SMALLWOOD, 1982; VAN DER PIJL, 1982); e 4) Barocoria - dispersão realizada apenas pelo peso do diásporo e por ação da força gravitacional (VAN DER PIJL, 1982).

Este estudo teve como objetivo identificar as proporções entre as síndromes de dispersão e os tipos de frutos das espécies arbustivo-arbóreas encontradas em três áreas: nascente (Cerrado Rupestre), meio e foz (mata ciliar-Floresta Estacional Semidecidual ribeirinha) ao longo do rio Pindaíba, MT, visando testar a hipótese de que no trecho de vegetação aberta de Cerrado (nascente) predomina a anemocoria, enquanto na floresta ciliar (meio e foz), a zoocoria.

\section{MATERIALE MÉTODOS}

Áreas de estudo - A nascente do rio Pindaíba (1544'24,8"Se 52²3'42,7'W) caracteriza-se por um Cerrado rupestre e está localizada na Fazenda Novo Horizonte, Município de Barra do Garças, MT.

O meio e a foz do rio Pindaíba são caracterizados comoáreas de mata ciliar-Floresta Estacional Semidecidual ribeirinha. O trecho do meio ( $14^{\circ} 53^{\prime} 01,8^{\prime \prime}$ S e 52 00 '33,8” W) localiza-se na Fazenda Nova Canaã, Município de Araguaiana, MT, enquanto a foz, onde o rio Pindaíba encontra o rio das Mortes, está localizada no Município de Nova Xavantina, MT, distante cerca de $80 \mathrm{~km}$ do centro da cidade, na Fazenda Pontal, nas coordenadas $14^{\circ} 27^{\prime} 20,4$ "'S e 5143'06,0"'W.

As três áreas de estudo estão compreendidas dentro do Bioma Cerrado. Foram selecionadas para este estudo porque estavam bem preservadas, representavam estágio sucessional avançado e sem sinais de perturbação antrópica recente, embora tivessem os entornos ocupados por pastagem. Com base em dados da Estação Meteorológica de Nova Xavantina, MT, o clima da região de estudo é classificado como Tropical Chuvoso de Savana (Aw) pelo sistema de Köppen, com duas estações bem marcadas: inverno seco de maio a setembro; e verão chuvoso de outubro a abril (ZILMER et al., 2007; IVANAUKAS et al., 2008).

A coleta de diásporos e as observações de campo foram realizadas durante o período de dois anos, para cobrir duas vezes todas as estações do ano e, dessa forma, amostrar o maior número possível de síndromes. Em cada trecho do rio, uma área de aproximadamente 1,0 ha foi visitada quinzenalmente. Em cada visita, as observações e os registros de campo foram realizados durante um período de seis horas, através de caminhadas aleatórias por toda a área.

A identificação das síndromes de dispersão foi realizada por meio de consultas à literatura e comparação dos diásporos com material depositado nas carpotecas dos herbários NX (UNEMAT - Campus Universitário de Nova Xavantina) e UB (Universidade de Brasília). Os diásporos anemocóricos e os tipos de frutos foram classificados de acordo com Barroso et al. (1999), Oliveira e Moreira (1992), Pinheiro e Ribeiro (2001) e Gottsberger e Sillberbauer-Gottsberger (1983). A identificação das espécies também foi realizada por meio de consulta à literatura e comparação com material depositado nos herbários NX e UB. A classificação das espécies nas respectivas famílias botânicas seguiu o sistema APG II (2003).

Para testar a hipótese de que as proporções de anemocoria eram maiores no trecho de Cerrado Rupestre (nascente do rio), e as de zoocoria eram na mata ciliar (meio e foz), utilizou-se o teste Qui-quadrado ( $\chi^{2}$ ) (ZAR, 1999), em nível de significância de 5\%, calculado através do software Bioestatística Usando R (BEASLEY, 2004). 


\section{RESULTADOS}

Trecho da nascente - No levantamento florístico realizado na nascente do rio Pindaíba foram amostradas 72 espécies pertencentes a 33 famílias (Tabela 1). Dentre as espécies arbustivo-arbóreas amostradas, 55,6\% (N $=40)$ eram zoocóricas, $43 \%(\mathrm{~N}=31)$ anemocóricas e apenas $1,4 \%(\mathrm{~N}=1)$ autocóricas.

As proporções observadas de autocoria foram desconsideradas para o cálculo do $\chi^{2}$, por serem muito pequenas, uma vez que esse teste requer frequências esperadas maiores do que cinco para amostras $\leq 40$ (VIEIRA, 2003). Assim, o resultado do teste mostrou que não havia diferença significativa entre as proporções de zoocoria e anemocoria na nascente $\left(\chi^{2}=0,8889\right.$; $\mathrm{gl}=1 ; p=0,3458)$.

Nas espécies anemocóricas houve diferentes proporções entre os tipos de diásporos: autogiro-rotativo ocorreu em $31,2 \%(\mathrm{~N}=10)$, autogiro em $28,2 \%(\mathrm{~N}=9)$, planador em $25 \%(\mathrm{~N}=8)$ e flutuante em $15,6 \%(\mathrm{~N}=5)$ das espécies.

Entre as 72 espécies listadas na área da nascente foram encontrados 19 tipos diferentes de frutos; entre estes, cápsula loculicida foi o tipo mais frequente, ocorrendo em $20 \%(\mathrm{~N}=15)$ das espécies. Os tipos de menor ocorrência foram cápsula septicida e folículo, ambos presentes em 5,3\% (N=4) das espécies amostradas. Os demais tipos de frutos encontrados na nascente totalizaram $69,4 \%(\mathrm{~N}=52)$.

Trecho do meio - No levantamento florístico realizado no meio do rio Pindaíba foram encontradas 77 espécies, distribuídas em 36 famílias (Tabela 1). Foram registradas $85,7 \%(\mathrm{~N}=66)$ de espécies zoocóricas, 11,7\%
$(\mathrm{N}=9)$ anemocóricas, $1,3 \%$ de autocóricas $(\mathrm{N}=1)$ e uma espécie permaneceu sem identificação da síndrome de dispersão. O resultado do $\chi^{2}$ mostrou que as proporções de zoocoria e anemocoria foram significativamente diferentes entre si $\left(\chi^{2}=47,1013 ; \mathrm{gl}=1 ; p=6,741 \times 10^{-12}\right)$.

$\mathrm{Na}$ área do meio do rio Pindaíba, as espécies anemocóricas apresentaram os seguintes tipos de diásporos: autogiro-rotativo, $\operatorname{com} 55,5 \%(\mathrm{~N}=5)$, autogiro com $22,3 \%$ $(\mathrm{N}=2)$ e planador e flutuante com $11,1 \%(\mathrm{~N}=1)$ cada.

Entre as 77 espécies amostradas no trecho meio do rio foram encontrados 27 tipos diferentes de frutos, sendo os com maior ocorrência: drupas, que representaram $15 \%(\mathrm{~N}=12)$; legume-nuculânio, 6,2\% $(\mathrm{N}=5)$; e núcula, $5 \%(\mathrm{~N}=4)$. Os demais tipos de frutos representaram $73,8 \%(\mathrm{~N}=59)$ do total.

Trecho da foz - Na foz do rio Pindaíba foram amostradas 80 espécies, distribuídas em 38 famílias (Tabela 1). Foram registradas $77,5 \%(\mathrm{~N}=62)$ de espécies zoocóricas, $20 \%(\mathrm{~N}=16)$ anemocóricas e apenas $2,5 \%(\mathrm{~N}=2)$ autocóricas. $\mathrm{O}$ resultado do teste $\chi^{2}$ (desconsiderando-se a barocoria e autocoria) indicou que as proporções de zoocoria e anemocoria eram significativamente diferentes $\left(\chi^{2}=25,8049\right.$; $\mathrm{gl}=1$; e $p=3,777 \times 10^{-7}$ ).

Entre os diásporos anemocóricos, verificaram-se as seguintes proporções: autogiro em $42,1 \%(\mathrm{~N}=8)$, planador em $31,6 \%(\mathrm{~N}=6)$, autogiro-rotativo em $21 \%(\mathrm{~N}=4)$ e helicóptero em 5,3\% ( $\mathrm{N}=1)$ das espécies.

Foram encontrados 23 tipos de frutos, em que drupa esteve presente em 16,8\% ( $\mathrm{N}=14)$, enquanto nuculânio, bacídeo e bacáceo, em apenas 5,9\% $(\mathrm{N}=5)$ das espécies amostradas. Todos os demais tipos de frutos ocorreram juntos em 44,1\% ( $\mathrm{N}=37)$ das espécies.

Tabela 1 - Espécies arbustivo-arbóreas encontradas na nascente, meio e foz do Rio Pindaíba, MT, com seus respectivos tipos de fruto e síndromes de dispersão. Nas.- Nascente; Cáps. Loc.- Cápsula Loculicida; Cáps. Sep.- Cápsula Septifraga; Cáps. Mista - Cápsula Mista \& *- Observação pessoal; M. Mon.- Múltiplo Monocárpico; M. Cup.Múltiplo Cupuliforme.

Table 1 - Shrubs-tree species found at the source, middle zone and estuary of River Pindaíba, MT, with their respective fruit types and dispersal syndromes. - Nas. - Source, Cáps. Loc. - Loculicidal capsule, Caps. Sep. - Septifragal capsule; Caps. Mista - Mixed Capsule \& *- Personal observation; M. Mon. - Multiple Monocarpic M. Cup Cupuliform Multiple.

\begin{tabular}{|c|c|c|c|c|c|}
\hline \multirow[t]{2}{*}{ Família / espécie } & \multirow[t]{2}{*}{ Dispersão } & \multicolumn{3}{|c|}{ Local } & \multirow[t]{2}{*}{ Tipo de Fruto } \\
\hline & & Nas. & Meio & Foz & \\
\hline $\begin{array}{l}\text { ANACARDIACEAE } \\
\text { Tapirira guianensis Aubl. }\end{array}$ & Zoocoria & & $\mathrm{X}$ & & Drupa \\
\hline
\end{tabular}


Tabela 1 - Cont.

Table 1 - Cont.

\begin{tabular}{l} 
Família / espécie \\
\hline ANNONACEAE \\
Annona coriacea Mart. \\
Duguetia marcgraviana Mart. \\
Guatteria coriacea R.E.Fr. \\
Guatteria rigida Ruiz.\& Pav. \\
Unonopsis lindmanii R.E.Fr. \\
Xylopia amazonica R.E.Fr. \\
Xylopia aromatica Lamb. \\
Xylopia sp.
\end{tabular}

\section{APOCYNACEAE}

Aspidosperma macrocarpon Mart.

Aspidosperma subincanum Mart.

Aspidosperma tomentosum Mart.

Aspidosperma sp.

Hancornia especiosa Gomes.

Himatanthus obovatus (Müll.Arg.) Woodson

Himatanthus sucuuba (Sprunce.ex.Müll.Arg) Woodson

Forsteronia rufa Mull Arg

\section{ARALIACEAE}

Schefflera macrocarpa (Seem) DC.Frodin.

\section{ARECACEAE}

Arecaceae NI

Astrocaryum vulgare Mart.

Attalea phalerata Mart.ex.Spreng.

Syagrus petraea (Mart.) Becc.

Syagrus sp.1

Syagrus sp.2

\section{ASTERACEAE}

Eremanthus cf. goyazensis (Garder) Sch.Bip.

Eremanthus sp.

\section{BIGNONIACEAE}

Arrabidaea cinnamomea (DC) Sandw.

Arrabidaea sp.

Tabebuia aurea Benth \& Hoock

Handroanthus serratifolius (Vahl) S. O. Grose

\section{BURSERACEAE}

Protium heptaphyllum (Aubl) Marchand.

Protium pilosissimum Engl.

Protium spruceanum (Benth) Engl.

Protium unifoliolatum Engl.

CHRYSOBALANACEAE

Couepia grandiflora (Mart.\&Zucc) Benth.\&Hook.f.

Couepia sp.

Hirtella glandulosa Spreng.

Hirtella gracilipes (Hook.F.) Prance.

Licania apetala (E.Mey.) Fritsch.

Licania gardneri (Hook.f.) Fritsch.
Dispersão

\begin{tabular}{lll} 
& Local & \\
Nas. & Meio & Foz \\
\hline
\end{tabular}

Tipo de Fruto
Múltiplo

Estrobiliforme

Sincarpo

Múltiplo

Multiplo livre

Apocarpo

Apocarpo

Apocarpo

Apocarpo

Múltiplo

Múltiplo

Múltiplo

Múltiplo

Bacóide

Cáps.Loc.

Cáps.Loc.

emocoria

Anemocoria

Zoocoria

Zoocoria

X

Folículo

Nuculânio

Anemocoria

Zoocoria

Zoocoria

Zoocoria

Zoocoria

Zoocoria

Anemocoria

Anemocoria

Anemocoria

Anemocoria

Anemocoria

Anemocoria

Zoocoria

Zoocoria

Zoocoria

Zoocoria

Zoocoria

Zoocoria

Zoocoria

Zoocoria

Zoocoria

Zoocoria
X

X

Aquênio

Drupa

Drupa

Drupa

Drupa

Drupa

Aquênio

Aquênio

Cáps.Loc.

Cáps.Loc.

Cáps.Loc.

Cáps.Loc.

Filotrimídio

Filotrimídio

Filotrimídio

Filotrimídio

X

Drupa

Drupa

Drupa

Drupa

Drupa Drupa

R. Árvore, Viçosa-MG, v.33, n.6, p.1051-1061, 2009 
Tabela 1 - Cont.

Table 1 - Cont

Família / espécie

Dispersão

\begin{tabular}{ccc}
\multicolumn{3}{c}{ Local } \\
\hline Nas. & Meio & Foz \\
\hline$X$ &
\end{tabular}

Tipo de Fruto

Licania humilis Cham.\&Schlecht.

Licania minutiflora (Sagot.) Frisch.

Licania kunthiana Hook.f.

CLUSIACEAE

Calophyllum brasiliense Cambess.

Garcinia brasiliensis Mart.

Kielmeyera coriacea Mart.

Kielmeyera rubriflora Cambess

Vismia sp.

\section{COMBRETACEAE}

Buchenavia tomentosa Eichler.

\section{CONNARACEAE}

Connarus suberosus Planch.

Rourea induta Planch.

COCHLOSPERMACEAE

Cochlospermum orinocense (Kunth.) Steud.

DICHAPETALACEAE

Tapura amazonica Poepp.

DILLENIACEAE

Davilla elliptica A.St.-Hill.

Davilla nitida (Vahl.) Kubitzki.

\section{EBENACEAE}

Diospyros xavantina Sothers

ELAEOCARPACEAE

Sloanea cf. guianensis (Aubl.) Benth.

Sloanea sinemariensis Aublet.

Sloanea terniflora (Sessé \& Moc.ex.DC.) Standl.

\section{ERYTHROXYLACEAE}

Erythroxylum suberosum A.St.-Hill.

Erythroxylum sp.1

Erythroxylum sp.2

Erythroxylum sp.3

Erythroxylum sp.4

\section{EUPHORBIACEAE}

Chaetocarpus echinocarpus (Baill.) Ducke.

Mabea fistulifera Mart.

Mabea pohliana (Benth) Müll. Arg.

Maprounea guianensis Aubl.

Sebastiana cf. brasiliensis Spreng.

\section{FABACEAE}

Acosmium nitens (Vogel) Yakovlev

Anadenanthera macrocarpa (Benth.) Brenan.

Anadenanthera peregrina (L.) Speg.

Andira cuyabensis Benth.

Andira sp.

Apuleia leiocarpa (Vogel) J.F.Macbr.

Bauhinia cf. cupulata Benth.

Bowdichia virgilioides Kunth.

Copaifera langsdorfii Desf. L.

Dimophandra mollis Benth.

Zoocoria
Zoocoria
Zoocoria
Zoocoria
Zoocoria
Anemocoria
Anemocoria
Zoocoria
Zoocoria
Zoocoria
Zoocoria

Anemocoria

$\mathrm{X}$

$\mathrm{X}$

$\mathrm{X}$

X $\mathrm{X}$

Drupa

Drupa

Drupa

\section{$\mathrm{X}$}

Drupa

$\mathrm{X}$

Bacóide

Cáps.Loc.

Cáps.Loc.

Campomane-soídio

\section{Drupa \\ Folículo \\ Folículo}

Cáps.Mista.

Zoocoria

X

Nuculânio

Zoocoria

X

Folículo

Zoocoria

X

Folículo

Zoocoria

X X

X $\quad \mathrm{X}$

Campomanesoídeo

Zoocoria

Zoocoria

Zoocoria

$\mathrm{X}$

Zoocoria

Zoocoria

Zoocoria

Zoocoria

Zoocoria

Zoocoria

Zoocoria

Zoocoria

Zoocoria

Zoocoria

X

X

Cáps.Loc.

Cáps.Loc.

Cáps.Loc.

Drupa

Drupa

Drupa

Drupa

Drupa

Cáps.Sép.

Esquizocarpo

Cáps.Loc.

Cáps.Loc.

Esquizocarpo

Anemocoria $\quad X \quad X \quad$ Legume Samarideo

Anemocoria

Anemocoria

Zoocoria

Zoocoria

Anemocoria

Autocoria

Anemocoria

Zoocoria

Zoocoria

$\mathrm{X}$

Legume

Legume

Drupa

Bacídeo

Legume Samarídeo Legume

Legume Samarídeo Legume

Legume Nuculânio

Enterolobium contortisiliquum (Vell.) Morong. 
Tabela 1 - Cont.

Table 1 -Cont.

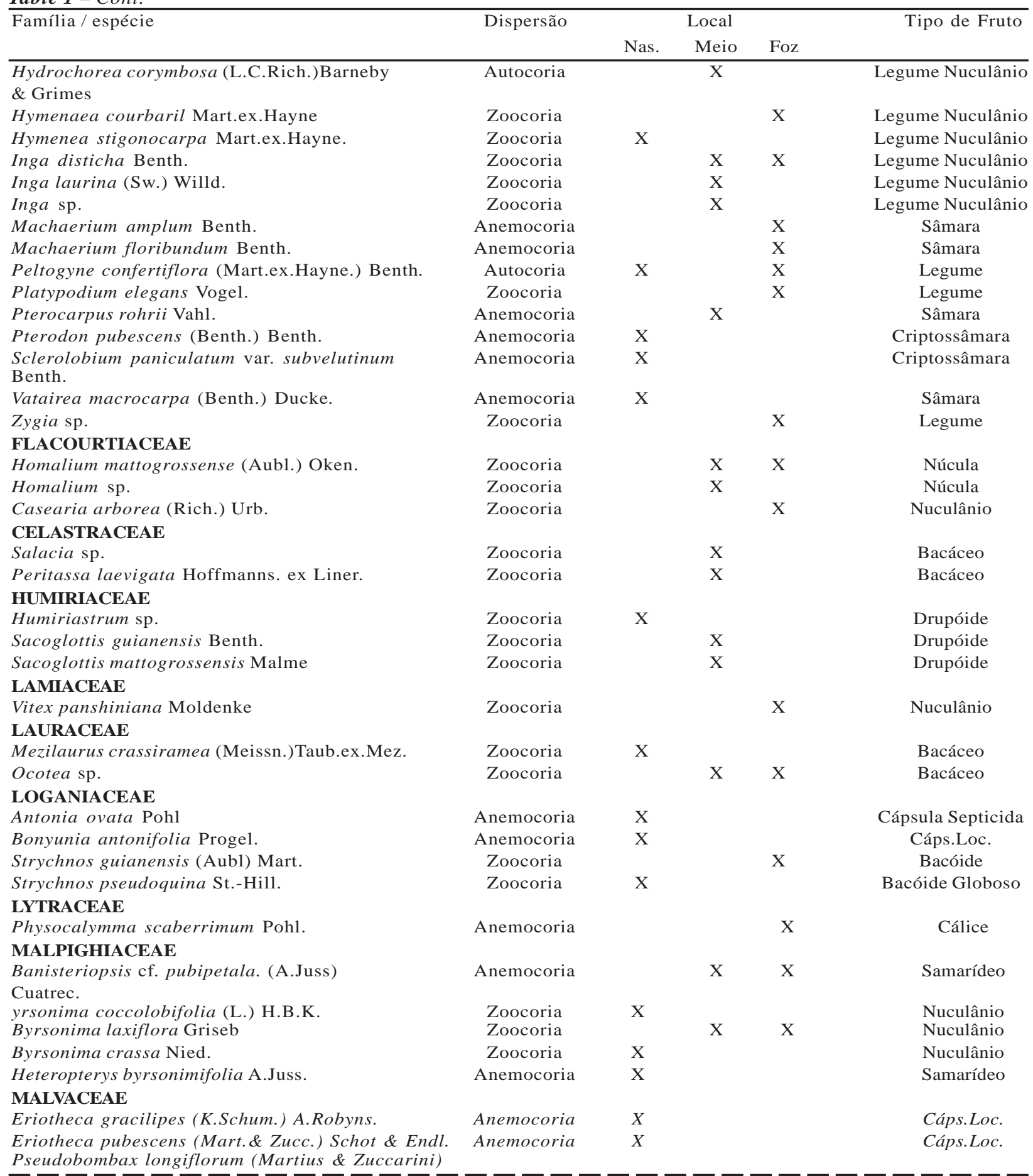

Continua...

Continued...

R. Árvore, Viçosa-MG, v.33, n.6, p.1051-1061, 2009 
Tabela 1 - Cont.

Table 1-Cont.

Família / espécie

Dispersão

\begin{tabular}{ccc}
\multicolumn{3}{c}{ Local } \\
\hline Nas. & Meio & Foz
\end{tabular}

Tipo de Fruto

A. Robyns

Guazuma tomentosa Kunth.

Helicteres sacarolha A.St.-Hil. A. Juss. \&

Cambess.

\section{MELASTOMATACEAE}

Chaetostoma sp.

Henrietella ovata Long.

Macairea radula (Bonpl.) DC.

Miconia albicans (Sw.) Triana.

Miconia holosericea (L.) Sleumer.

Miconia tomentosa (Rich.)D.Don.ex.DC.

Miconia sp.

Mouriri apyranga Sprunce.ex.Triana

Mouriri elliptica Mart.

MELIACEAE

Guarea guidonea (L.) Sleumer.

MENISPERMACEAE

Abuta grandifolia (Mart.) Sandwith.

Abuta sellowiana Eicher.

\section{MORACEAE}

Brosimum sp.

Ficus guianensis Desv.ex.Ham.

Pseudolmedia laevigata Trécul.

Sorocea guilleminiana Gaudich.

MYRISTICACEAE

Virola urbaniana Warb.

MYRSINACEAE

Cybianthus detergens Mart.

MYRTACEAE

Eugenia florida DC.

Eugenia egensis DC.

Eugenia sp.

Gomidesia sp.

Myrcia amazonica DC.

Myrcia camapuanensis N.Silveira.

Myrcia canescens Berg.

Myrcia deflexa (Poir) DC.

Myrciaria dubia (Kunth.) McVaugh.

Myrcia multiflora (Lam.) DC.

Myrcia variabilis Mart.ex.Benth.\&Hook.f.

Myrcia sp.

Psidium paraense O.Berg.

NYCTAGINACEAE

Guapira noxia (Netto) Lundell.

Neea theifera Orsted.

OCHNACEAE

Ouratea castaneifolia (DC.) Eng1.

Ouratea hexasperma (A.St.-Hill.) Baill.

Ouratea spectabilis (Mart.) Engl.

Zoocoria

Anemocoria

Anemocoria
Zoocoria
Anemocoria
Zoocoria
Zoocoria
Zoocoria
Zoocoria
Zoocoria
Zoocoria

Zoocoria

Zoocoria

Zoocoria

Zoocoria

Zoocoria

Zoocoria

Zoocoria

Zoocoria

Zoocoria

Zoocoria

Zoocoria

Zoocoria

Zoocoria

Zoocoria

Zoocoria

Zoocoria

Zoocoria

Zoocoria

Zoocoria

Zoocoria

Zoocoria

Zoocoria

Zoocoria

Zoocoria

Zoocoria

Zoocoria

Zoocoria

\section{X}

$X$

Cáps. Loc.

Cáps. Septicida

Velatídio

Bacídio

Velatídio

Bacídio

Bacídio

Bacídio

Bacídio

Bacídio

Bacídio

Cáps.Loc.

Drupa

Hipocrepiforme

M. Mon.

M. Cup.

Núcula

Núcula

Folículo

Drupa

Bacóide

Bacóide

Bacáceo

Bacóide

Bacáceo

Bacáceo

Bacáceo

Bacáceo

Bacáceo

Bacáceo

Bacáceo

Bacáceo

Solanídio

Núcula

Núcula

Múltiplo Livre Múltiplo Livre Múltiplo Livre

OLACACEAE

Continua...

Continued.. 
Tabela 1 - Cont.

Table 1 - Cont

Família / espécie

Dispersão

Nas.

Local

Meio

Dulacia inopiflora (Miers.) Kuntze.

OPILIACEAE

Agonandra brasiliensis Miers. ex. Benth. \& Hook.f. Zoocoria PENTAPHYLACACEAE

Ternstroemia candolleana Wawra.

PROTEACEAE

Roupala montana Aubl.

\section{RUBIACEAE}

Alibertia sp.

Alibertia sp. 1

Alibertia sp.2

Amaioua sp.

Ferdinandusa elliptica Pohl.

Genipa americana $\mathrm{L}$.

Psychotria carthaginensis Jacq.

Psychotria cf. platypodiana Müll. Arg.

Psychotria sp.

Psychotria sp. 1

Psychotria sp.2

Rhandia cf. armata (Sw.) DC.

SAPINDACEAE

Matayba guianensis Aubl.

Serjania sp.

SAPOTACEAE

Micropholis gardneriana (DC.) Pierre.

Micropholis venulosa (Mart. \& Eichler) Pierre.

Pouteria macrophylla (Mart.) Bacheni.

Pouteria ramiflora (Mart.) Radlk.

Pouteria torta (Mart.) Radalk.

Pouteria sp.

Zoocoria

X

X

X

Drupa

Anemocoria

X

X

Globoso

Zoocoria

Zoocoria

Zoocoria

Zoocoria

Anemocoria

Zoocoria

Zoocoria

Zoocoria

Zoocoria

Zoocoria

Zoocoria

Zoocoria

Zoocoria

Anemocoria

Zoocoria

Zoocoria

Zoocoria

Zoocoria

Zoocoria

Zoocoria

Zoocoria

Simarouba amara Aubl.

SIPARUNACEAE

Siparuna guianensis Aubl.

Sess \& Mart.

VELLOZIACEAE

Vellozia cf. flavicans Mart.ex.Schut.f.

VOCHYSIACEAE

Callisthene fasciculata (Spreng.) Mart.

Qualea grandiflora Mart.

Qualea multiflora Mart.

Qualea parviflora Mart.

Salvertia convallariodora A.St.-Hill.

Vochysia divergens Pohl.

Vochysia gardneri Mart.

Vochysia hankeana Mart.

Vochysia rufa Mart.
Zoocoria

Zoocoria

Anemocoria

Anemocoria

Anemocoria

Anemocoria

Anemocoria

Anemocoria

Anemocoria

Anemocoria

Anemocoria

Anemocoria
X

X

$x$

X

X

$\mathrm{X}$

$\begin{array}{ll} & \\ & X \\ X & \\ X & \\ X & \\ X\end{array}$

X

Folículo

Bacóide

Bacídeo

Bacídeo

Anfissarcideo

Cáps.Sep.

Anfissarsídio

Nuculânio

Nuculânio

Nuculânio

Nuculânio

Nuculânio

Bacóide

Cáps.Loc.

Sâmara

Bacídeo

Bacídeo

Bacóide

Bacóide

Bacóide

Bacóide

Múltiplo

M. Cup.

Drupa

Cápsula

Cáps.Loc.

Cáps.Loc.

Cáps.Loc.

Cáps.Loc.

Cáps.Loc.

Cáps.Loc.

Cáps.Loc.

Cáps.Loc.

Cáps.Loc. 


\section{DISCUSSÃO}

Trecho da nascente - Em áreas abertas, em geral, espera-se encontrar maior número de espécies anemocóricas (OLIVEIRA e MOREIRA, 1992). Neste estudo, embora a nascente do rio Pindaíba seja caracterizada como um Cerrado Rupestre, portanto uma fitofisionomia savânica (RIBEIRO e WALTER, 1998), não houve diferença significativa entre as proporções de espécies zoocóricas e anemocóricas.

Alguns autores acreditam que a anemocoria é a síndrome mais importante em ambientes abertos (FENNER, 1985; OLIVEIRA e MOREIRA, 1992) até porque um dossel fechado diminui consideravelmente a ação do vento. No entanto, as proporções similares de anemocoria e zoocoria na nascente do rio Pindaíba possivelmente são explicadas pela proximidade da área estudada com manchas de vereda e mata de galeria; ambientes reconhecidamente tidos como refúgio, abrigo e fonte de alimento para a fauna (VAN DER PIJL, 1982; MARINHO FILHO e GASTAL, 2000), o que pode ter influenciado a composição das espécies amostradas.

Martins et al. (2004), em áreas de Cerrado stricto sensu em Itirapina, SP, encontraram maior proporção de espécies zoocóricas $(58,6 \%)$ do que de anemocóricas $(28,6 \%)$, enquanto as espécies autocóricas também apresentaram as menores proporções $(12,8 \%)$. Já Vieira et al. (2002), estudando áreas de Cerrado stricto sensu no Brasil central e áreas de savanas amazônicas, relataram que a zoocoria ocorreu com maior frequência tanto no Cerrado quanto nas áreas de savanas amazônicas estudadas, com proporções entre 51,1 e $68,3 \%$ de espécies zoocóricas, contra 26,7 a $46,7 \%$ de espécies anemocóricas, respectivamente. Os resultados aqui encontrados indicam a importância da zoocoria para essa área. Uma das hipóteses para explicar as vantagens da dispersão por animais é a da colonização e dispersão dirigida, ou seja, a zoocoria permite a dispersão de sementes maiores e, ao mesmo tempo, é mais direcionada do que a anemocoria (HOWE e SMALLWOOD, 1982).

Cabe, contudo destacar que as proporções similares de anemocoria e zoocoria nesse trecho do rio mostram que ambas as síndromes são importantes na manutenção das populações de plantas, e a anemocoria possibilita deposição mais aleatória de grande quantidade de propágulos. Além disso, a anemocoria envolve pouco investimento energético por parte da planta-mãe na formação dos propágulos, não dependendo, portanto, de animais dispersores (VAN DER PIJL, 1982). No entanto, ainda não está claro até que ponto a quantidade de sementes dispersas pelo vento compensa a perda de exatidão e direcionamento conseguidos com outros mecanismos de dispersão, como a zoocoria, por exemplo (VAN DER PIJL, 1982; HOWE e SMALLWOOD, 1982).

O tipo de fruto mais frequente na nascente foi cápsula loculicida, o qual é seco e deiscente, de acordo com Barroso et al. (1999). Uma vez que a dispersão de sementes geralmente está relacionada a melhores condições ambientais para a liberação dos diásporos e estabelecimento das plântulas (VAN DER PIJL, 1982), a maioria das espécies anemocóricas e autocóricas possuem frutos secos, deiscentes, que frutificam na estação seca (VAN DER PIJL, 1982).

Meio e Foz - Na mata ciliar do meio foram encontrados $85,7 \%$ de espécies zoocóricas e apenas $11,7 \%$ de anemocóricas, enquanto na mata ciliar da foz 77,5\% das espécies eram zoocóricas, $20 \%$ anemocóricas e 2,5\% autocóricas. Como esperado, as proporções de zoocoria nas matas ciliares do meio e da foz são significativamente diferentes das proporções de anemocoria.

Esees resultados são semelhantes aos encontrados por Meira Neto et al. (2003) em mata de galeria no Município de Viçosa, MG. Os referidos autores constataram que $12,5 \%$ eram anemocóricas, e as demais $(87,5 \%)$ eram endozoocóricas, revelando um padrão similar ao encontrado neste estudo.

Em uma compilação de dados entre 19 matas de galeria do Distrito Federal, Pinheiro e Ribeiro (2001) também observaram que o número de espécies dispersas por animais é superior em todas as áreas comparadas, tendo como resultado geral $72 \%$ de zoocoria, $24 \%$ de anemocoria e apenas 3 e $1 \%$ de espécies autocóricas e barocóricas, respectivamente.

Resultados semelhantes também foram observados em outras formações vegetais, como em uma Floresta Estacional Semidecidual no Paraná, onde $84,4 \%$ das espécies eram zoocóricas (MIKICH e SILVA, 2001), bem como em uma floresta de brejo na região de Campinas, SP, onde foram registradas $57 \%$ de espécies zoocóricas, $27 \%$ de anemocóricas e $16 \%$ de autocóricas (SPINA et al., 2001). Talora e Morellato (2000) encontraram $89 \%$ de espécies zoocóricas e apenas $10 \%$ de anemocóricas em uma área de floresta no Parque Estadual da Serra do Mar, em São Paulo.

R. Árvore, Viçosa-MG, v.33, n.6, p.1051-1061, 2009 
Formações florestais, como as matas ciliares que ocorrem em manchas mais ou menos isoladas em locais com maior disponibilidade de água e,ou, protegidas do fogo (RIBEIROe WALTER, 2001), permitem a manutenção de uma fauna característica, que busca refúgio, alimento eágua(MARINHO-FILHOeGASTAL, 2000), oque favorece a zoocoria nesses ambientes.

Em ambientes florestais, a cobertura arbórea dificulta a ação do vento, o que acaba limitando a dispersão de espécies anemocóricas no sub-bosque (OLIVEIRA e MOREIRA, 1992), tanto que em florestas tropicais a anemocoria está associada a árvores emergentes e lianas (OLIVEIRAe MOREIRA, 1992). Assim, o estabelecimento de indivíduos nessas condições requer sementes maiores e com grande quantidade de material de reserva (VAN DER PIJL, 1982). Nesse caso, em ambientes florestais, frutos com sementes grandes podem favorecer a capacidade de sobrevivência das plântulas em condições de baixa luminosidade e alta competição por recursos (PRIMACK, 1987; BUEN e ORNELAS, 2001), mas também apresentam maior gasto de energia e, consequentemente, dependem mais dos agentes dispersores (zoocoria).

Os resultados encontrados no meio e foz do rio Pindaíba mostram que o tipo de fruto mais frequente foi drupa. De acordo com Barroso et al. (1999), esse tipo de fruto está relacionado a um epicarpo e mesocarpo com consistência carnosa e com um só pirênio no seu interior. Pinheiro e Ribeiro (2001) consideraram que há relação entre a morfologia dos frutos e a guilda de frugívoros que utiliza esse recurso, indicando que a variedade de tipos de frutos encontrada na mesma síndrome reflete a riqueza de estratégias utilizadas pelas plantas para atrair os dispersores.

A hipótese levantada neste estudo foi que, em áreas abertas (nascente), a proporção de espécies anemocóricas em relação às zoocóricas seria maior do que em ambientes fechados (meio e foz), onde predominaria a zoocoria. No entanto, não houve diferença significativa nas proporções de espécies anemocóricas e zoocóricas na nascente, o que pode ser atribuído ao fato de que, mesmo se tratando de área de Cerrado, a proximidade de matas ciliares possibilitou a ocorrência de espécies com dispersão zoocórica. Nos trechos meio e foz, as proporções de zoocoria foram maiores. Esse padrão parece ser comum em regiões tropicais, ou seja, há convergência entre as áreas para uma estrutura com alta proporção de zoocoria, proporções intermediárias de anemocoria e baixa ou nenhuma de autocoria e barocoria (PINHEIRO e RIBEIRO, 2001).

\section{REFERÊNCIAS}

ANGIOSPERM PHYLOGENY GROUP II. - APG II. An update of the Angiosperm Phylogeny Group classificationfor the orders and families of flowering plants: APG II. Botanical Journal of the Linnean Society, v.141, p.399-436, 2003.

BARROSO, G. M. et al. Frutos e sementes: morfologia aplicada à sistemática de dicotiledôneas. Viçosa,MG: Universidade Federal de Viçosa, 1999.

BEASLEY, C. R. Bioestatística usando R. Universidade Federal do Pará. 2004. Disponível em: http://www.rproject.org/other-docs.html.

BUEN, L. L.; ORNELAS, J. F. Seed dispersal of the Psittacanthus schiedeanus by birds in central Vera Cruz, México. Biotropica, v.33, n.3, p.487-494, 2001.

FENNER, M. Seed ecology. London: Chapman and Hall, 1985.

\section{GOTTSBERGER, G; SILLBERBAUER-}

GOTTSBERGER, I. Dispersal and distribuition in the Cerrado vegetation of Brazil. Sonderband des

Natuwissenchaftichen Vereins in Hamburg, v.7, p.315-352, 1983.

GRAHAM, C. H. et al. Seed dispersal effectiveness by two bulbuls on Masea lanceolata, an African montane forest tree. Biotropica, v.27, n.4, p.479486, 1995.

HOWE, H. F.; SMALLWOOD, J. Ecology of seed dispersal. Ann. Rev. Ecol. Syst., v.13, p.201228, 1982.

IVANAUSKAS, N. M.; MONTEIRO, R.; RODRIGUES, R. R. Classificação fitogeográfica das florestas do Alto Rio Xingu. Acta Amazônica, v.38, n.3, p.387-402, 2008.

MARINHO FILHO, J.; GASTAL, M. L. Mamíferos da matas ciliares do Brasil Central. In: RODRIGUES, R. R. (Org.), Matas ciliares: estado atual do conhecimento. São Paulo: FAPESP, 2000. p.209-221.

MIKICH, S. B.; SILVA, S. M. Composição florística e fenologia das espécies zoocóricas de remanescentes de Floresta Estacional Semidecidual no Centro-Oeste do Paraná, Brasil. Acta Botanica Brasílica, v.15, n.1, p.89-113, 2001. 
MARTINS, F. Q. et al. Síndromes de dispersão no componente arbustivo-arbóreo em fragmentos de cerrado, no município de Itirapina, São Paulo. In: SANTOS, F. A. M.; MARTINS, F. R.; TAMASHIRO, J. Y. (Orgs.). Relatórios de projetos desenvolvidos na disciplina NE211 Ecologia de campo II - do Programa de Pós-graduação em Ecologia, IB, UNICAMP. Campinas: UNICAMP, 2004.

MEIRA NETO J. A. A. et al. Origem, sucessão e estrutura de uma floresta de galeria periodicamente alagada em Viçosa-MG. Revista Árvore, v.27, n.4, p.561-574, 2003.

OLIVEIRA P. E. A. M.; MOREIRA, A. G. Anemocoria em espécies de cerrado e mata de galeria de Brasília - DF. Revista Brasileira de Botânica, v.15, n.2, p.163-174, 1992.

PETERNELLI, E. F.; DELLA LUCIA, T. M. C.; MARTINS, S. V. Espécies de formigas que interagem com as sementes de Mabea fistulifera Mart. (Euphorbiaceae). Revista Árvore, v.28, n.5, p.733-738, 2004.

PINHEIRO, F.; RIBEIRO, J. R. Síndromes de dispersão de sementes em matas de galeria do Distrito Federal. In: RIBEIRO, J. F.; FONSECA, C. E. L.; SOUSA-SILVA, J. C. (Eds.). Cerrado: caracterização e recuperação de matas de galeria. Brasília: Embrapa, 2001.p.335-361.

PRIMACK, R. B. Relationships among flowers, fruits, and seeds. Ann. Rev. Ecol. Syst., v.18, p.409430, 1987.

RIBEIRO, J. F.; WALTER, B. M. T. Fitofisionomias do Bioma Cerrado. In: SANO, S. M.; ALMEIDA, S. P. (Eds.). Cerrado: ambiente e flora. Brasília: Embrapa, 1998. p.89-152.
RIBEIRO, J. F.; WALTER, B. T. M. As matas de galeria no contexto do bioma Cerrado. In: RIBEIRO, J. F.; FONSECA, C. E. L.; SOUSASILVA, J. C. (Eds.). Cerrado: caracterização e recuperação de matas de galeria. Brasília: Embrapa Cerrados, 2001. p.29-45.

SPINA, A. P.; FERREIRA, W. M.; LEITÃO-FILHO, H. F. Floração, frutificação e síndrome de dispersão de uma comunidade de Floresta de Brejo na região de Campinas (SP). Acta Botanica Brasílica, v.15, n.3, p.349-368, 2001.

TALORA, D. C.; MORELLATO, P. C. Fenologia de espécies arbóreas em uma floresta de planície litorânea do sudeste do Brasil. Revista Brasileira de Botânica, v.23, n.1, p.13-26, 2000.

VIEIRA, D. L. M. et al. Síndromes de dispersão de espécies arbustivo-arbóreas em Cerrado Sensu Stricto do Brasil Central e Savanas Amazônicas. Revista Brasileira de Botânica, v.5, n.2, p.215-220, 2002.

VIEIRA, S. Bioestatística: tópicos avançados. Rio de Janeiro: Campus, 2003.

van der PIJL L. Principles of dispersal in higher plants. 3.ed. New York: Springer Verlag, 1982.

ZAR, J. H. Biostatistical analysis. 4.ed. New Jersey: Prentice Hall, 1999.

ZILMER, T. A.; VARELLA, R. F.; ROSSETE, A. N. Avaliação de algumas características físícoquimícas das águas do ribeirão Salgadinho, Nova Xavantina, MT. Holos Environment, v.7, n.2., p/126-138, 2007. 
